\title{
Diversité des champignons endomycorhiziens de l'arganier et potentiel mycorhizogène des sols rhizosphériques des arganeraies du Sud-Ouest marocain \\ (n)
}

Imane OUALLAL ${ }^{1}$

Younes ABBAS ${ }^{2}$

Sara ECH-CHEDDADI ${ }^{1}$

Mohamed OUAJDI ${ }^{3}$

Moussa OUHADACH ${ }^{1}$

Houda EL YACOUBI ${ }^{1}$

Benaissa KeRDOUH ${ }^{3}$

Younes EL GoumI ${ }^{4}$

Atmane RoCHDI ${ }^{1}$

${ }^{1}$ Université Ibn Tofaïl Unité d'agrophysiologie et phytobiotechnologie BP242, Kénitra

Maroc

2 Université Sultan Moulay Slimane Faculté polydisciplinaire Laboratoire polyvalent R\&D BP 592, Béni Mellal

Maroc

${ }^{3}$ Centre de recherche forestière BP 763, Rabat

Maroc

${ }^{4}$ Agence Nationale des Plantes Médicinales et Aromatiques Laboratoire de Phyto-Biotechnologie Taounate

Maroc

\section{Auteur correspondant /}

Corresponding author:

Imane Ouallal -

imaneouallal@gmail.com

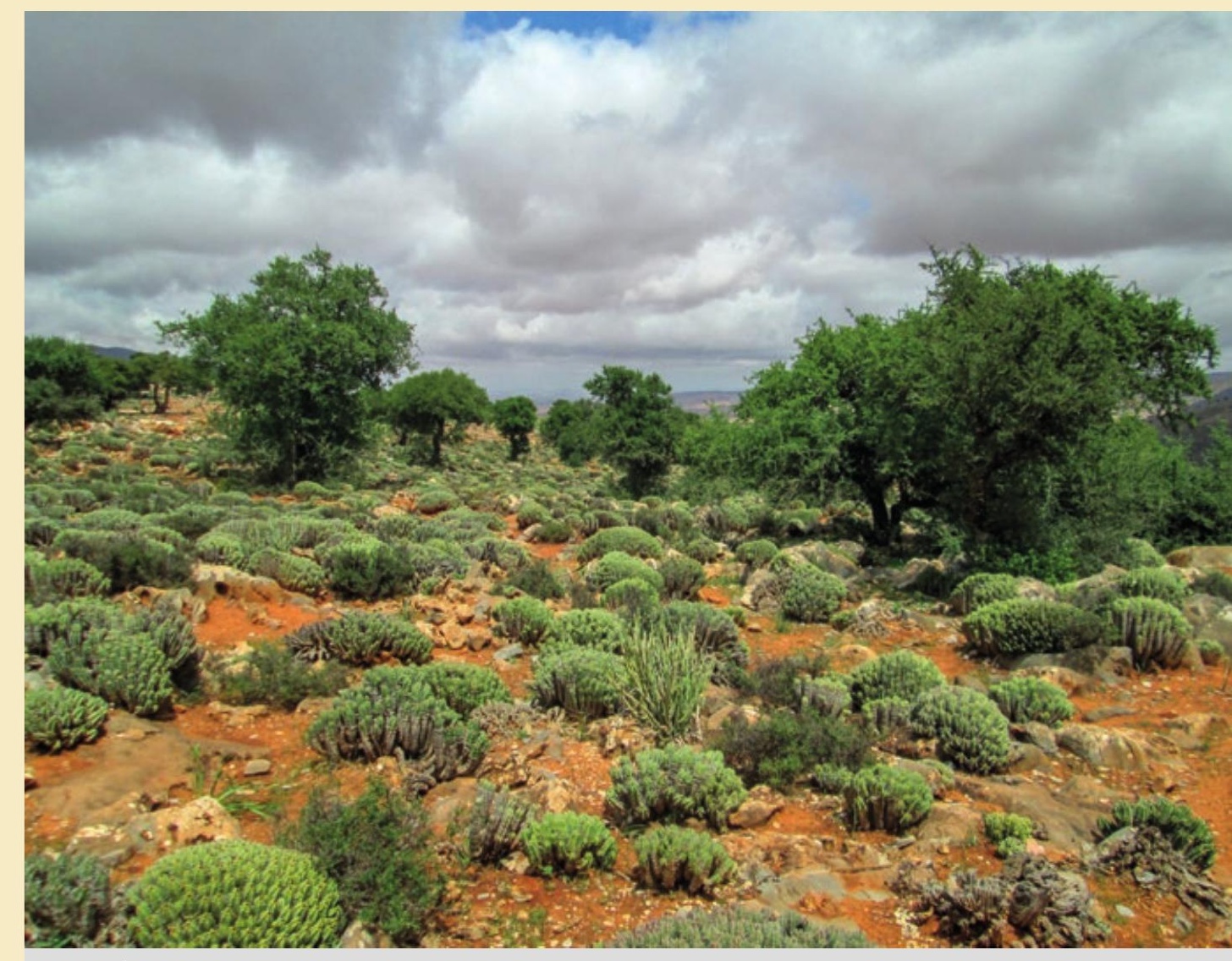

Photo 1.

Vue d'une partie de l'Arganeraie de Lekhssas. Photo I. Ouallal.

Doi : 10.19182/bft2018.338.a31678 - Droit d'auteur (c) 2018, Bois et Forêts des Tropiques @ Cirad - Date de soumission : 14 septembre 2017 ; date d'acceptation : 14 juin 2018 ; date de publication : $1^{\text {er }}$ décembre 2018.

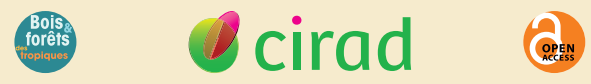

(c) $(1) \Theta$ Attribution-NoDerivatives 4.0 International (CC BY-ND 4.0)
Citer l'article / To cite the article

Ouallal I., Abbas Y., Ech-cheddadi S., Ouajdi M., Ouhadach M., El Yacoubi H., Benaissa K., El Goumi Y., Rochdi A., 2018. Diversité des champignons endomycorhiziens de l'arganier et potentiel mycorhizogène des sols rhizosphériques des arganeraies du Sud-Ouest marocain. Bois et Forêts des Tropiques, $n^{\circ} 338$ : 73-86. Doi : https://doi.org/10.19182/bft2018.338. a31678 


\section{RÉSUMÉ}

Diversité des champignons

endomycorhiziens de l'arganier

et potentiel mycorhizogène des sols

rhizosphériques des arganeraies

du Sud-Ouest marocain

L'arganier, Argania spinosa, est une espèce endémique du Maroc, structurant des formations forestières remarquables et façonnant un terroir agricole original. Si son statut mycorhizien a été largement étudié, la diversité mycorhizienne associée et son efficacité, en tenant compte du lien avec les contextes pédoclimatiques et la diversité des territoires de l'arganier, demeure méconnue. Aussi, neuf arganeraies du SudOuest marocain ont fait l'objet d'investigations afin, d'une part, d'explorer la diversité des communautés de champignons mycorhiziens associés à cet arbre dans diverses situations édapho-climatiques et, d'autre part, d'évaluer le potentiel mycorhizogène des environnements rhizosphériques, en vue de les exploiter comme sources d'inocula. L'examen microscopique des racines de l'arganier confirme la mycotrophie de l'espèce et sa dépendance aux champignons mycorhiziens arbusculaires (CMA), quel que soit le site prospecté. Les spores de CMA isolées des sols rhizosphériques des arganeraies étudiées restent de faible densité. Les corrélations entre les différents morphotypes sont très hautement significatives au sein du genre Glomus. Dans certains sites, une corrélation positive apparaît entre la densité de spores et l'intensité de mycorhization des racines. Une corrélation négative se présente entre le potentiel mycorhizien des neuf sols étudiés et les propriétés chimiques des sols. Ces résultats suggèrent que d'autres facteurs édaphiques (propriétés biologiques des sols, par exemple) ou le degré de perturbation des sols des arganeraies, lié aux usages agricoles et au pâturage, pourraient influencer fortement le processus de mycorhization de l'arganier. Il en est ainsi de la performance des spores mycorhiziennes, ce qui conduit à devoir éviter de contre-sélectionner des CMA inefficients en matière de survie et de croissance de l'arganier, qu'il s'agisse de production en pépinières forestières ou de réhabilitation en milieu naturel.

Mots-clés : Argania spinosa, champignons mycorhiziens, potentiel infectieux mycorhizogène, rhizosphère, biodiversité, Maroc.

\section{ABSTRACT}

\author{
Diversity of endomycorrhizal fungi \\ on argan tree roots and potential for \\ mycorrhizal development in the soil \\ rhizosphere of argan stands in south- \\ western Morocco
}

Argania spinosa, the argan tree, is endemic to Morocco and a key species in woodlands of outstanding interest that shape a unique agricultural landscape. While its mycorrhizal status has been extensively studied, the diversity and efficiency of associated mycorrhiza, taking the pedo-climate relationship into account as well as the diversity of argan habitats, is largely unknown. For this reason, this study investigated nine argan woodlands in south-western Morocco in order to (i) explore the diversity of mycorrhizal fungi communities associated with argan trees in different soil and climate conditions and (ii) assess the potential for the development mycorrhizal communities in rhizosphere environments, with a view to their use as sources of inocula. Microscope examinations of argan roots confirmed the mycotrophic character of the species and its dependence on arbuscular mycorrhizal fungi (AMF) in all the sites prospected. The AMF spores isolated from the soil rhizospheres in the argan stands studied are found in low densities. The correlations between the different morphotypes are very highly significant in the Glomus genus. For some sites, a positive correlation appears between the density of spores and the degree of root mycorrhization, while the mycorrhizal potential of the nine soils studied is negatively correlated with the chemical properties of the soils. These results suggest that other edaphic factors (e.g. biological soil properties), or soil disturbance in the argan stands due to farming or grazing, could strongly influence the mycorrhization process. This is the case with the performance of the mycorrhizal spores, which indicates a need to avoid counter-selecting AMF that are not effective in terms of argan tree survival and growth, whether the trees are grown in forestry nurseries or in the natural environment for woodland rehabilitation.

Keywords: Argania spinosa, mycorrhizal fungi, mycorrhizal infection potential, rhizosphere, biodiversity, Morocco.

\author{
Diversidad de hongos endomicorrizas \\ del argán y potencial micorrizógeno \\ de los suelos rizosféricos de los bosques \\ de argán del sudoeste marroquí
}

El argán, Argania spinosa, es una especie endémica de Marruecos que estructura formaciones forestales destacables y conforma una tierra agrícola original. Aunque su estado micorrícico ha sido ampliamente estudiado, la diversidad micorrícica asociada y su eficacia, teniendo en cuenta la relación con los contextos pedoclimáticos y la diversidad de los territorios del argán, continúa sin conocerse. Así, nueve bosques de argán del sudoeste marroquí han sido objeto de investigación para (i) explorar la diversidad de las comunidades de hongos micorrícicos asociados a este árbol en diversas situaciones edafoclimáticas, y (ii) evaluar el potencial micorrizógeno de los entornos rizosféricos, en vistas a una explotación como fuentes de inocula. El examen microscópico de las raíces del argán confirma la microtrofia de la especie y su dependencia de los hongos micorrizas arbusculares (HMA), independientemente de donde se prospecte. Las esporas de HMA aisladas en suelos rizosféricos de los bosques de argán estudiados continúan siendo de baja densidad. Las correlaciones entre diferentes morfotipos son muy altamente significativas en el seno del género Glomus. En ciertos lugares, aparece una correlación positiva entre la densidad de esporas y la intensidad de micorrización de las raíces. Se encuentra una correlación negativa entre el potencial micorrícico de los nueve suelos y las propiedades químicas de los suelos. Estos resultados sugieren que otros factores edáficos (ej. propiedades biológicas de los suelos) o el grado de alteración de los suelos de los bosques de argán, relacionado con los usos agrícolas y el pastoreo, podrían influir fuertemente en el proceso de micorrización del argán. Este rendimiento de las esporas micorrícicas implica que se debe evitar contra-seleccionar HMA ineficientes en materia de supervivencia y de crecimiento del argán, tanto si se trata de producción en viveros forestales como de rehabilitación en medio natural.

Palabras clave: Argania spinosa, hongos micorrizas, potencial infeccioso, micorrícico, rizosfera, biodiversidad, Marruecos. 


\section{Introduction}

L'arganier (Argania spinosa), « arbre providentiel » du Sud-Ouest marocain, fait partie du patrimoine universel de l'Unesco depuis 1998. Cette espèce endémique du Sud atlantique du Maroc représente un cas emblématique du patrimoine génétique marocain. L'arganier fait l'objet depuis assez longtemps d'une exploitation à des fins de recherche scientifique fondamentale et de développement de produits divers (alimentaires, pharmaceutiques et cosmétiques) ayant trait aux utilités multiples de l'arganeraie (intérêt écologique, vocation fruitière, utilisations pastorale et forestière).

$\mathrm{Au}$ Maroc, l'arganeraie couvre une aire approximative de 828000 hectares (M'Hirit et al., 1998). L'arganier est adapté aux régions arides et semi-arides où il joue un rôle très important dans la lutte contre la désertification et l'érosion. Cependant, en plus des effets du changement climatique global (succession et allongement des périodes de sécheresse), les écosystèmes à arganier sont menacés par la pression anthropique croissante (pression démographique, ramassage systématique des fruits, labours et développement de l'agriculture irriguée, prélèvement du bois, abroutissement et surpâturage aérien par les chèvres). Cette pression provoque une régression des arganeraies, aussi bien en superficie qu'en densité, ainsi qu'un vieillissement des populations en raison d'une faible régénération (Nouaim, 1991). Durant le dernier siècle, environ 50 \% de son territoire a été perdu (600 ha/an) et sa densité moyenne est passée de 100 à 30 arbres par hectare (Abourouh, 2007). L'arganeraie, qui constitue un milieu naturel exceptionnel et un terroir agricole original, est donc fortement menacée.

Les terres des arganeraies marocaines sont de la sorte souvent perturbées, ce qui peut conduire à une faiblesse du potentiel mycorhizogène des sols (Benabid, 2000). Par ailleurs, les champignons mycorhiziens à arbuscules (CMA) présents naturellement dans les agrosystèmes sont à prendre en considération pour une agriculture plus durable, qui tienne compte de l'écologie et de la biologie du sol (Van der Heijden, 2001). L'inoculation des plantes par les CMA peut non seulement faciliter leur installation (Smith et Read, 2008 ; Alguacil, 2011), mais aussi améliorer les propriétés physico-chimiques et biologiques des sols (Rillig et Mummey, 2006 ; Schmid et al., 2008).

Dans le cadre du Plan Maroc Vert, le Maroc s'est engagé à entreprendre des réexamens radicaux en faveur d'une meilleure valorisation et une gestion durable de ses ressources naturelles. En outre, la sauvegarde des espèces indigènes les mieux adaptées à leur milieu nécessite d'utiliser des biotechnologies impliquant la multiplication de plants sélectionnés sur des critères de production et de résistance aux maladies ou aux stress de l'environnement, en accordant une importance particulière à la gestion du système racinaire (Abourouh, 1992). En effet, la biodiversité et les liens fonctionnels entre les organismes sont les bases de la stabilité, de la productivité et de la résilience des écosystèmes (Tilman et Downing, 1994). Les mycorhizes participent de ces liens en offrant aux plantes des avantages bénéfiques à leur survie. L'utilisation de micro-organismes bénéfiques pour la régénération des espèces forestières est une voie raisonnée de réduction des apports d'intrants et de préservation des écosystèmes forestiers (Nouaim et Chaussod, 1997). Selon Malusá et al. (2014), les inoculations microbiennes et fongiques constituent des sources respectueuses de l'environnement, en faveur de la production d'engrais organiques.

Selon Kenny et al. (2009), il n'y a pas d'étude exhaustive de la diversité des CMA associés à l'arganier. Il nous apparaissait donc judicieux de caractériser la diversité mycorhizienne associée à cet arbre, en tenant compte du lien avec les contextes pédoclimatiques (semi-aride, aride et saharien) et la diversité des territoires de l'arganier (continental, côtier, montagne et plaine). L'objectif de cette étude était, d'une part, d'explorer, au sein des arganeraies du Sud-Ouest marocain, la diversité des communautés de champignons mycorhiziens associés à l'arganier dans différentes situations édapho-climatiques et, d'autre part, d'évaluer le potentiel mycorhizogène des environnements rhizosphériques.

\section{Matériel et méthodes}

\section{Sites d'étude}

Neuf sites (Safi, Essaouira, Admine, Amskroud plaine, Amskroud montagne, Imouzzer, Argana, Lekhssas et Bouizakarne) d'arganiers situés dans le semi-aride et l'aride méditerranéen ont été prospectés. Ces stations représentent différentes conditions écogéographiques des arganeraies du Sud-Ouest marocain (tableau I). Les principales espèces associées à l'arganier dans les différents sites prospectés sont Olea maroccana, Thymus satureioides, Polygala balansae, Periploca angustifolia, Euphorbia beaumierana, Euphorbio echini, Euphorbia regis jubae, Acacia gummifera, Hesperolaburnum platycarpum et Hammada scoparia.

\section{Échantillonnage pour le prélèvement de sols et de racines}

Le prélèvement des échantillons a été effectué en février 2013 dans les neuf arganeraies. Notre stratégie d’échantillonnage a consisté à éliminer d'abord les racines des plantes accompagnatrices. Nous avons aussi considéré la distribution verticale des champignons avant de récolter nos échantillons.

Cinq arganiers adultes, représentatifs de l'aspect général des pieds de chaque station, ont été échantillonnés dans chaque site prospecté. Ensuite, six sous-échantillons d'environnements rhizosphériques ont été prélevés autour de chaque arbre, à plus de $20 \mathrm{~cm}$ de profondeur sachant qu'entre 20 et $40 \mathrm{~cm}$ on peut trouver les racines les plus fines susceptibles d'être mycorhizées, en prenant soin de ne pas abîmer les fines racines d'arganier qui sont été également récoltées. Les prélèvements de « sols rhizosphériques » de chaque arganier ont été mélangés, homogénéisés et placés dans un contenant en plastique afin d'obtenir un échantillon moyen composite (environ $2 \mathrm{~kg}$ ), représentatif de la rhizosphère de l'arbre échantillonné. 
Tableau I.

Caractéristiques géographiques et climatologiques des sites échantillonnés.

\begin{tabular}{|c|c|c|c|c|c|}
\hline Province & Sites & Coordonnées & $\begin{array}{l}\text { Altitude } \\
\text { (m) }\end{array}$ & $\begin{array}{l}\text { Température moyenne } \\
\text { annuelle }\left({ }^{\circ} \mathrm{C}\right)\end{array}$ & $\begin{array}{l}\text { Pluviométrie moyenne } \\
\text { annuelle }(\mathrm{mm})\end{array}$ \\
\hline Safi & Safi & $\mathrm{N}: 313700 \mathrm{~W}: 80000$ & 18 & 18,1 & 488,5 \\
\hline Essaouira & Essaouira & $\mathrm{N}: 313047 \mathrm{~W}: 94611$ & 21 & 17,3 & 343 \\
\hline \multirow[t]{4}{*}{ Agadir Ida-Outanane } & Admine & $\mathrm{N}: 335539 \mathrm{~W}: 93622$ & 59 & 20 & 243 \\
\hline & Amskroud plaine & $N: 303151$ W: 91942 & 261 & 18,3 & 254 \\
\hline & Amskroud montagne & $N: 303650$ W: 92351 & 636 & 14 & 500 \\
\hline & Imouzzer & $N: 304048$ W: 92858 & 729 & 14 & 500 \\
\hline Taroudant & Argana & $\mathrm{N}: 304644 \mathrm{~W}: 90733$ & 735 & 20,9 & 500 \\
\hline Sidi Ifni & Lekhssas & $N: 394047$ W: 97258 & 950 & 21,7 & 149 \\
\hline Guelmim & Bouizakarne & $\mathrm{N}: 291034 \mathrm{~W}: 94251$ & 690 & 24,9 & 120 \\
\hline
\end{tabular}

Parallèlement, pour chaque arganier, des échantillons de racines fines, susceptibles d'être mycorhizées et plus facilement observables sous microscope, ont été récupérés dans un flacon contenant une solution de glycérol-éthanol-eau distillée $(1 \mathrm{v} / 1 \mathrm{v} / 1 \mathrm{v})$. Tous les échantillons ont été numérotés, mis en obscurité, ramenés au laboratoire et conservés au réfrigérateur à $4^{\circ} \mathrm{C}$ pour être utilisés durant les deux mois qui suivent.

\section{Analyses physico-chimiques du sol}

Les principales propriétés physico-chimiques des échantillons de sols ont été analysées en utilisant les méthodes conventionnelles. Le $\mathrm{pH}$ a d'abord été mesuré dans de l'eau distillée, puis dans une solution normale de $\mathrm{KCl}$, en recourant à la méthode électrométrique à électrode en verre. L'analyse granulométrique des sols par densimétrie a été déterminée par la méthode de Mériaux (1954). Le phosphore assimilable a été dosé par colorimétrie selon la méthode d'Olsen et al. (1954). L'extraction a été assurée par une solution alcaline d'hydrogénocarbonate à $\mathrm{pH} 8,5$ pendant une heure à $20^{\circ} \mathrm{C}$. Le carbone organique a été dosé par la méthode d'Anne (1945) qui consiste à oxyder la matière organique du sol par un mélange de bichromate de potassium en milieu sulfurique à chaud, jusqu'au dégagement de $\mathrm{CO}_{2}$.

\section{Critères d'évaluation de la mycorhization}

L'évaluation des critères de la mycorhization a été réalisée, pour chaque échantillon, sur 30 fragments de racines fines colorées selon la méthode de Philips et Hayman (1970) et montées dans du lacto-glycérol (ce qui permet la conservation, limite l'évaporation et améliore l'indice de réfraction optique selon Lachapelle, 2004) sur trois lames contenant dix fragments chacune. Sous microscope optique, les racines ont été examinées soigneusement sur toute leur longueur afin de noter les structures mycorhiziennes. Les paramètres notés étaient la fréquence et l'intensité de mycorhization des racines, ainsi que les teneurs en arbuscules et en vésicules des endomycorhizes à l'intérieur de l'écorce racinaire (Trouvelot et al., 1986).

\section{Extraction et identification des spores mycorhiziennes}

Cent grammes de sol sec ont été utilisés pour l'isolement des spores par la technique de tamisage humide selon la méthode de Gerdeman et Nicolson (1963). Les spores fongiques récupérées ont d'abord été observées sous loupe binoculaire ( $x 40$ ), puis sous microscope ( $x 100$ ). Les spores ont été considérées comme viables si elles avaient un contenu clair au microscope optique, avec une paroi intacte. Elles ont été classées en fonction principalement de leurs caractéristiques morphologiques (taille, couleur, forme, consistance, structure de la paroi et de l'attachement de l'hyphe suspenseur), puis quantifiées. Un calcul simple a ensuite permis d'exprimer le résultat en nombre de spores par $100 \mathrm{~g}$ de sol sec.

La classification des morphotypes au niveau du genre et, si possible, au niveau de l'espèce a principalement reposé sur les caractéristiques morphologiques, en se basant sur quelques clés d'identification (Koske et Tessier, 1983 ; Morton et Benny, 1990 ; Brundrett et al., 1996), et en se référant aux descriptions fournies par la collection internationale de champignons mycorhiziens à arbuscules et vésicules de l'INVAM ${ }^{1}$.

Les indices suivants ont été évalués :

- la densité de spores, correspondant au nombre de spores par $100 \mathrm{~g}$ de sol sec ;

- l'indice de Shannon, qui permet d'exprimer la diversité en prenant en compte le nombre d'espèces et l'abondance des individus au sein de chacune de ces espèces :

$\mathrm{H}^{\prime}=-\sum_{i=1}^{\mathrm{s}} \mathrm{p}_{i} \ln \mathrm{p}_{i}$

avec $\mathrm{p}_{i}$ : abondance proportionnelle ou pourcentage d'importance de l'espèce, $\mathrm{p}_{i}=\mathrm{n}_{i} / \mathrm{N} ; \mathrm{S}$ : nombre total d'espèces ; $\mathrm{n}_{i}$ : nombre d'individus d'une espèce dans l'échantillon ; $\mathrm{N}$ : nombre total d'individus de toutes les espèces dans l'échantillon ;

- l'indice d'équitabilité de Piélou (E), qui est la régularité de la distribution des espèces :

$\mathrm{E}=\mathrm{H}^{\prime} / \log _{2} \mathrm{~S}$

avec $S$ : nombre total d'espèces ; H' : indice de Shannon.

1 International Culture Collection of (Vesicular) Arbuscular Mycorrhizae : http://invam.caf.wvu.edu 


\section{Potentiel mycorhizogène des sols}

Le potentiel mycorhizogène (PM) d'un sol exprime la richesse du sol en propagules aptes à générer les mycorhizes. Il représente non seulement la population des champignons mycorhiziens présents dans le sol sous forme de spores, de mycéliums et de morceaux de mycorhizes, mais aussi la capacité de cette population à initier la formation d'associations mycorhiziennes. Il est considéré comme un indicateur biologique de l'état de dégradation d'un sol (Mosse, 1986).

Le PM des échantillons des sols rhizosphériques récupérés à partir des différentes stations a été déterminé par la technique de dilution (Sieverding, 1991) en utilisant l'orge (Hordeum vulgare L.) comme plante mycotrophe.

Après trois mois de croissance, le système racinaire des plants d'orge de chaque pot a été recueilli et lavé sous un filet d'eau du robinet, puis éclairci et coloré selon la méthode de Philips et Hayman (1970) afin de visualiser les structures mycorhiziennes. Des échantillons de racines ont été montés entre lame et lamelle et observés au microscope optique pour l'évaluation de toute trace de mycorhizes.

La présence de structures mycorhiziennes a été notée au niveau des cinq répétitions (pots). Les échantillons des systèmes radiculaires montrant un point d'infection (pénétration d'un hyphe dans la racine) ont été considérés comme mycorhizés (Sanon, 2005) et marqués du signe (+) ; par contre, ceux dont les racines n'étaient pas infectées ont été marqués du signe (-). Le nombre le plus probable (NPP) de propagules par unité de sol, qui est une estimation par maximum de vraisemblance du nombre de propagules infectieuses par poids de sol testé (Cochran, 1950), a été calculé conformément à Sieverding (1991) selon la formule :

$$
\log \Omega=\text { X.log } \mathrm{a}-\mathrm{K}
$$

avec $\Omega$ : nombre de propagules infestées; $\mathrm{X}$ : nombre de pots infestés ; $X$ : total de pots infestés / nombre de répétitions par dilution $(23 / 5=4,6)$; $Y$ : nombre de niveaux de dilution $-x$, soit, dans notre cas, $Y=6-x$; a : facteur de dilution (soit 4 dans notre cas) ; K était lue dans la table proposée par Alexander (1965) et Fisher et Yates (1970).

\section{Analyse statistique}

Les données ont été analysées par JMP SAS Pro software $^{2}$. La normalité de la distribution des données a été analysée par le test de Shapiro-Wilk ; la normalité a été assurée par la transformation $\operatorname{Arcsin} \sqrt{x}$, puis les données ont été statistiquement traitées par l'analyse de la variance (ANOVA) et le test de Fisher (modèle linéaire par moindres carrés standard). Les moyennes des sites, des morphotypes et leur interaction ont été comparées par le test HSD (Honestly Significant Difference) de Tukey au risque d'erreur de 0,05.

\section{Résultats}

\section{Caractéristiques physico-chimiques des sols rhizosphériques de l'arganier}

La distribution des fractions minérales (tableau II) a varié entre les différentes zones ; les sols de Lekhssas et Amskroud plaine étaient les plus riches en argile (31\% et $24 \%$ ), le sol de Safi était le plus riche en limon (71\%) et les sols d'Essaouira et Imouzzer étaient les plus riches en sable (74\% et $72 \%$ ).

Les sols de Safi et Amskroud plaine présentaient les teneurs les plus élevées en matière organique et carbone total. Cependant, les sols prélevés des neuf sites présentaient des teneurs en phosphore assimilable toujours faibles, quel que soit le site considéré.

Le $\mathrm{pH}$ des échantillons rhizosphériques prélevés dans les différents sites de l'arganier était légèrement alcalin $(7,12$ à 7,62).

2 JMP®, Version 〈13〉. SAS Institute Inc.

Tableau II.

Propriétés physico-chimiques des sols rhizosphériques de l'arganier.

\begin{tabular}{|c|c|c|c|c|c|c|c|c|c|c|}
\hline \multirow[t]{2}{*}{ Sites } & \multirow{2}{*}{$\begin{array}{l}\text { Argile } \\
(\%)\end{array}$} & \multicolumn{2}{|c|}{ Limon (\%) } & \multicolumn{2}{|c|}{ Sable (\%) } & \multirow[t]{2}{*}{ pH eau } & \multirow[t]{2}{*}{$\mathrm{pH} \mathrm{KCl}$} & \multirow{2}{*}{$\begin{array}{c}\text { Carbone } \\
(\%)\end{array}$} & \multirow{2}{*}{$\begin{array}{c}\text { Matière } \\
\text { organique } \\
\text { (\%) }\end{array}$} & \multirow{2}{*}{$\begin{array}{l}\text { Phosphore } \\
\text { assimilable } \\
\text { (ppm) }\end{array}$} \\
\hline & & Fin & Grossier & Fin & Grossier & & & & & \\
\hline Safi & 0,8 & 18,4 & 52,20 & 20,15 & 8,45 & 7,25 & 6 & 8,563 & 14,729 & 51 \\
\hline Essaouira & 0,8 & 17,2 & 8,45 & 29,3 & 44,25 & 7,50 & 6 & 0,844 & 1,452 & 20 \\
\hline Admine & 11,6 & 28,6 & 16,6 & 18,55 & 24,65 & 7,55 & 5 & 1,326 & 2,282 & 20 \\
\hline Amskroud plaine & 23,6 & 9,7 & 4 & 25,2 & 37,5 & 7,12 & 5 & 7,719 & 13,277 & 54 \\
\hline Amskroud montagne & 0,8 & 22,13 & 14,37 & 13,6 & 49,1 & 7,20 & 4 & 1,447 & 2,489 & 43 \\
\hline Imouzzer & 12,6 & 11,7 & 4 & 18,45 & 53,25 & 7,62 & 2 & 3,859 & 6,638 & 24 \\
\hline Argana & 19,6 & 22,7 & 18,95 & 18,25 & 20,5 & 7,45 & 4 & 4,583 & 7,883 & 27 \\
\hline Lekhssas & 31,2 & 15,36 & 16,34 & 13,7 & 23,4 & 7,52 & 4 & 1,326 & 2,282 & 19 \\
\hline Bouizakarne & 13,6 & 21,6 & 10,5 & 20,5 & 33,8 & 7,43 & 6 & 1,085 & 1,867 & 5 \\
\hline
\end{tabular}




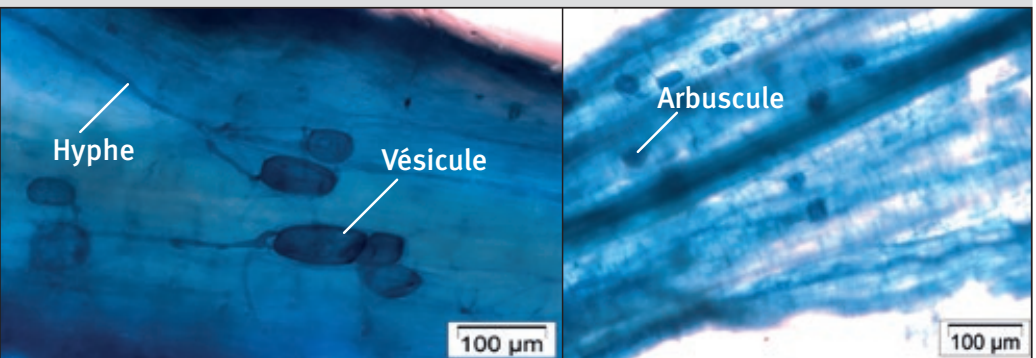

Figure 1.

Fragments mycorhizés de racine d'arganier et structures des endomycorhizes.
Par contre, pour les autres paramètres de mycorhization, il a été remarqué, d’un côté, un effet significatif du facteur site sur la teneur en vésicules ; d'un autre côté, même si l'ANOVA a montré que ce facteur présentait une source de variation pour la teneur en arbuscules, la comparaison des moyennes par le test de Tukey ne confirmait pas la significativité des différences des sites en teneur en arbuscules.

\section{Diversité et densité des spores de CMA dans les sols rhizosphériques de l'arganier}

\section{Mycorhization naturelle de l'arganier}

L'examen microscopique des fragments de racines de l'arganier a montré que tous les échantillons étaient densément colonisés par des champignons mycorhiziens et que l'organisation cytologique de ces mycorhizes était de type arbusculaire, avec présence de vésicules de différentes formes (globulaire, ovale et amorphe) et d'hyphes endomycorhizogènes (figure1).

La fréquence de mycorhization a été toujours très élevée (94 à 100\%) et ne variait pas significativement entre les sites (tableau III). De même, pour les intensités mycorhiziennes, malgré quelques différences quant aux taux observés, particulièrement dans les sites de Safi ainsi que de Essaouira et Bouizakarne, cette différence restait non significative.
Le nombre de morphotypes de spores, déterminés selon la forme, la couleur et la taille, a varié d'un site à un autre. En effet, la richesse spécifique a été de six espèces de spores dans les sites de Safi, Essaouira et Bouizakarne, cinq à Admine et Amskroud plaine, quatre à Amskroud montagne, Argana et Lekhssas, et enfin deux seulement dans le site d'Imouzzer. En analysant cette richesse spécifique en CMA dans les neuf sols rhizosphériques de l'arganier, nous avons déterminé seulement sept morphotypes (tableau IV).

Le nombre de spores isolées le plus élevé (207 spores par $100 \mathrm{~g}$ de sol sec) a été observé dans le site d'Amskroud plaine, suivi par celui de Bouizakarne (108 spores par $100 \mathrm{~g}$ de sol sec), mais il restait très faible au niveau des sols rhizosphériques d'Imouzzer, Essaouira et Argana (respectivement 19, 3 et 35,0 spores par $100 \mathrm{~g}$ de sol sec) (figure 2).

\section{Tableau III.}

Comparaison des moyennes d'effets des sites ( \pm erreur standard) sur la fréquence et l'intensité de mycorhization ainsi que la teneur en vésicules et arbuscules.

\begin{tabular}{|c|c|c|c|c|c|c|}
\hline \multirow[b]{2}{*}{ Sites } & \multicolumn{2}{|c|}{ Mycorhization } & \multicolumn{2}{|c|}{ Teneur } & \multicolumn{2}{|c|}{ Caractéristiques des sites } \\
\hline & $\begin{array}{l}\text { Fréquence } \\
(\%)\end{array}$ & $\begin{array}{c}\text { Intensité } \\
\text { (\%) }\end{array}$ & $\begin{array}{l}\text { Vésicules } \\
\text { (\%) }\end{array}$ & $\begin{array}{c}\text { Arbuscules } \\
\text { (\%) }\end{array}$ & $\begin{array}{l}\text { Densité } \\
\text { des arbres }\end{array}$ & $\begin{array}{l}\text { Usage } \\
\text { du site }\end{array}$ \\
\hline Admine & $94 \pm 4,00 \mathrm{a}$ & $14,39 \pm 5,85 a$ & $47,79 \pm 9,70 a b$ & $13,75 \pm 11,49 a$ & Moyenne & $\begin{array}{c}\text { Protection } \\
\text { (mise en défens) }\end{array}$ \\
\hline Amskroud M & $100 \pm 0,00 \mathrm{a}$ & $14,96 \pm 5,44 \mathrm{a}$ & $51,11 \pm 1,11 a b$ & $10,56 \pm 4,75 a$ & Moyenne & Pâturage \\
\hline Amskroud P & $100 \pm 0,00 \mathrm{a}$ & $28,83 \pm 5,27 a$ & $21,11 \pm 5,88 b$ & $4,17 \pm 3,00 a$ & Moyenne & Agricole \\
\hline Argana & $100 \pm 0,00 a$ & $13,06 \pm 4,76 a$ & $30,00 \pm 11,55 a b$ & $3,89 \pm 2,42 a$ & Moyenne & Pâturage \\
\hline Bouizakarne & $98 \pm 2,00 \mathrm{a}$ & $34,08 \pm 6,51 \mathrm{a}$ & $52,22 \pm 9,85 a b$ & $35,92 \pm 8,77 a$ & Faible & Pâturage \\
\hline Essaouira & $94 \pm 4,00 \mathrm{a}$ & $38,42 \pm 12,87 a$ & $39,21 \pm 4,61 a b$ & $2,70 \pm 1,15 a$ & Moyenne & Pâturage \\
\hline Imouzzer & $98 \pm 2,00 a$ & $27,76 \pm 10,58 a$ & $17,23 \pm 6,22 b$ & $28,07 \pm 17,06 \mathrm{a}$ & Moyenne & Pâturage \\
\hline Lekhssas & $100 \pm 0,00 \mathrm{a}$ & $26,94 \pm 8,18 a$ & $70,22 \pm 20,60 a$ & $1,33 \pm 1,33 a$ & Faible & Pâturage \\
\hline Safi & $100 \pm 0,00 \mathrm{a}$ & $41,34 \pm 10,25 \mathrm{a}$ & $31,11 \pm 4,84 a b$ & $3,33 \pm 1,67 a$ & Faible & Agricole \\
\hline Moyenne & $98,22 \pm 0,73$ & $26,64 \pm 2,89$ & $40,00 \pm 4,15$ & $11,52 \pm 3,15 a$ & & \\
\hline
\end{tabular}

Pour chaque paramètre, les moyennes connectées par la même lettre ne sont pas significativement différentes à $P=0,05$. Les valeurs sont présentées en moyenne \pm erreur standard. 
Tableau IV.

Comparaison des moyennes ( \pm erreur standard) d'effets des sites, des morphotypes et leurs interactions sur le nombre de spores.

\begin{tabular}{|c|c|c|c|c|c|c|c|c|}
\hline \multirow{2}{*}{$\begin{array}{l}\text { Paramètres } \\
\text { Sites }\end{array}$} & \multirow[b]{2}{*}{$\begin{array}{c}\text { Rhizophagus } \\
\text { aggregatus }\end{array}$} & \multirow[b]{2}{*}{$\begin{array}{l}\text { Funneliformis } \\
\text { constrictum }\end{array}$} & \multirow[b]{2}{*}{$\begin{array}{c}\text { Sclerocystis } \\
\text { sinuosa }\end{array}$} & \multirow{2}{*}{$\begin{array}{c}\text { Morphotypes } \\
\text { Rhizophagus } \\
\text { intraradices }\end{array}$} & \multirow[b]{2}{*}{$\begin{array}{c}\text { Rhizophagus } \\
\text { irregularis }\end{array}$} & \multirow[b]{2}{*}{$\begin{array}{l}\text { Scutellospora } \\
\text { sp1 }\end{array}$} & \multirow[b]{2}{*}{$\begin{array}{l}\text { Scutellospora } \\
\text { sp2 }\end{array}$} & \multirow[b]{2}{*}{$\begin{array}{c}\text { Moyenne } \\
\text { (sites) }\end{array}$} \\
\hline & & & & & & & & \\
\hline Admine & $1,60 \pm 0,40 f$ & $33,60 \pm 6,68 \mathrm{bcde}$ & $12,60 \pm 6,13$ cdef & $4,80 \pm 1,53 \mathrm{f}$ & $0,00 \pm 0,00 \mathrm{f}$ & $15,60 \pm 4,78 \mathrm{cdef}$ & $0,00 \pm 0,00 f$ & $9,74 \pm 2,36 b c$ \\
\hline Amskroud M & $0,00 \pm 0,00 \mathrm{f}$ & $18,00 \pm 1,26$ cdef & $17,00 \pm 6,77$ cdef & $5,20 \pm 2,24 f$ & $0,00 \pm 0,00 f$ & $6,00 \pm 3,48$ ef & $0,00 \pm 0,00 \mathrm{f}$ & $6,60 \pm 1,63 b c$ \\
\hline Amskroud P & $5,20 \pm 3,32 f$ & $34,80 \pm 9,22 \mathrm{bcd}$ & $18,20 \pm 2,22$ cdef & $109,20 \pm 26,23 a$ & $35,00 \pm 3,87 \mathrm{bcd}$ & $0,00 \pm 0,00 f$ & $0,00 \pm 0,00 \mathrm{f}$ & $28,91 \pm 7,12 a$ \\
\hline Argana & $0,00 \pm 0,00 \mathrm{f}$ & $18,20 \pm 7,11$ cdef & $15,00 \pm 3,51$ cdef & $2,80 \pm 1,59 f$ & $0,00 \pm 0,00 f$ & $0,80 \pm 0,37 f$ & $0,00 \pm 0,00 \mathrm{f}$ & $5,26 \pm 1,63 c$ \\
\hline Bouizakarne & $0,00 \pm 0,00 \mathrm{f}$ & $11,00 \pm 5,57$ cdef & $37,60 \pm 4,31 b c$ & $11,80 \pm 0,73$ cdef & $0,40 \pm 0,24 \mathrm{f}$ & $5,80 \pm 3,56$ ef & $0,00 \pm 0,00 \mathrm{f}$ & $9,51 \pm 2,36 b c$ \\
\hline Essaouira & $0,00 \pm 0,00 \mathrm{f}$ & $10,20 \pm 3,47$ cdef & $11,40 \pm 6,76$ cdef & $0,00 \pm 0,00 \mathrm{f}$ & $0,00 \pm 0,00 f$ & $0,00 \pm 0,00 f$ & $0,00 \pm 0,00 \mathrm{f}$ & $3,09 \pm 1,29 c$ \\
\hline Imouzzer & $0,00 \pm 0,00 \mathrm{f}$ & $4,40 \pm 0,51 \mathrm{f}$ & $0,00 \pm 0,00$ & $14,40 \pm 0,68$ cdef & $0,00 \pm 0,00 f$ & $0,00 \pm 0,00 \mathrm{f}$ & $0,00 \pm 0,00 \mathrm{f}$ & $2,69 \pm 0,87 c$ \\
\hline Lekhssas & $0,00 \pm 0,00 f$ & $5,60 \pm 2,71 \mathrm{f}$ & $19,40 \pm 2,23$ cdef & $24,00 \pm 5,32$ bcdef & $0,00 \pm 0,00 f$ & $0,00 \pm 0,00 \mathrm{f}$ & $0,40 \pm 0,40 \mathrm{f}$ & $7,06 \pm 1,83 b c$ \\
\hline Safi & $0,00 \pm 0,00 \mathrm{f}$ & $47,60 \pm 12,64 b$ & $6,80 \pm 2,15$ ef & $2,40 \pm 0,81 \mathrm{f}$ & $9,20 \pm 1,74$ def & $9,60 \pm 2,87$ def & $22,80 \pm 6,50$ bcdef & $14,06 \pm 3,24 b$ \\
\hline $\begin{array}{l}\text { Moyenne } \\
\text { (morphotypes) }\end{array}$ & $0,76 \pm 0,42 b$ & $20,38 \pm 2,92 \mathrm{a}$ & $15,33 \pm 1,98 a$ & $19,40 \pm 5,60 a$ & $4,96 \pm 1,71 b$ & $4,20 \pm 1,09 b$ & $2,58 \pm 1,26 b$ & \\
\hline
\end{tabular}

Pour chaque paramètre, les moyennes connectées par la même lettre ne sont pas significativement différentes à $P=0,05$.

Les valeurs sont présentées en moyenne \pm erreur standard.

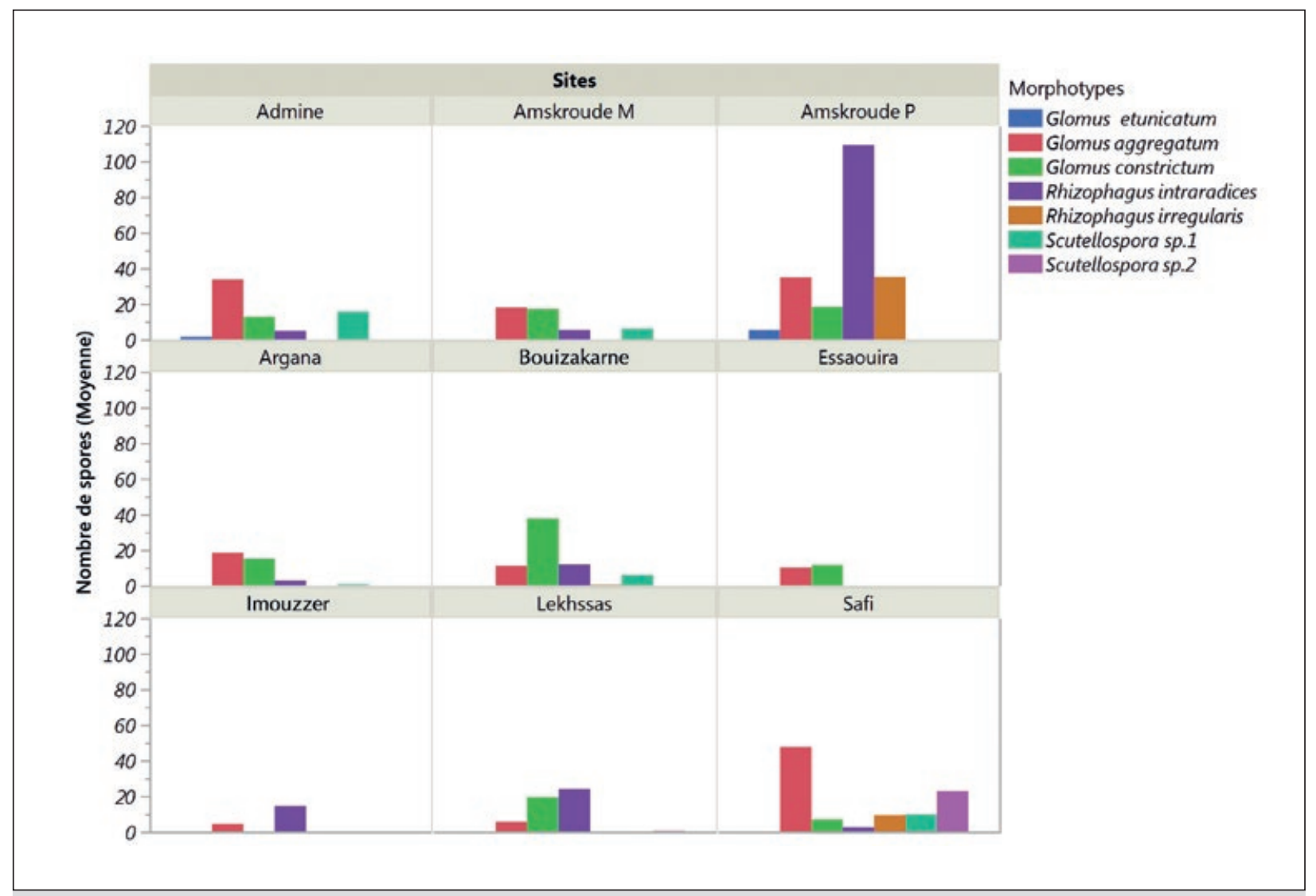

Figure 2.

Répartition du nombre de spores des sept morphotypes par site

(les données sont exprimées par la moyenne des valeurs). 


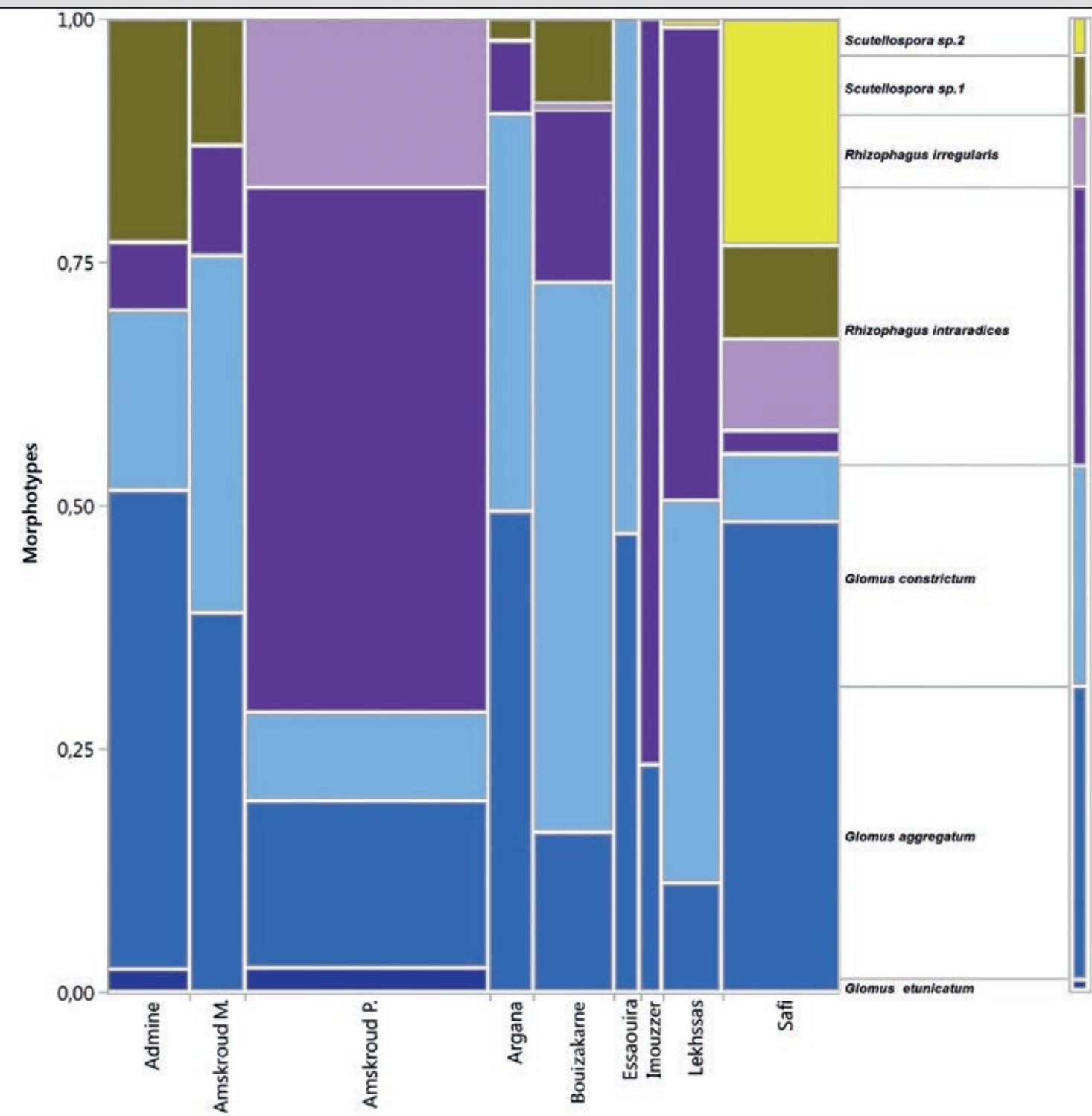

Sites

Figure 3.

Fréquence des morphotypes des spores de CMA en fonction des sites étudiés.

Les spores isolées appartenaient à l'ordre des Glomales, ici représenté par les familles Glomaceae et Gigasporaceae. L'analyse des caractéristiques morpho-anatomiques de cette communauté de spores a révélé la présence de deux genres (Glomus et Scutellospora) dont la répartition de leurs espèces isolées est présentée dans la figure 3. Le genre Glomus était représenté dans nos échantillons par cinq espèces (Rhizophagus aggregatus, Funneliformis constrictum, Sclerocystis sinuosa, Rhizophagus intraradices, Rhizophagus irregularis), et le genre Scutellospora par deux espèces (Scutellospora sp1 et Scutellospora sp2) (figure 4).
Le calcul de l'indice de diversité (Shannon) et celui d'équitabilité de Piélou (E) dans nos sites d'étude (figure 5) ont montré chacun une similarité d'abondance, à l'exception du site de Safi qui a montré une richesse spécifique plus élevée avec six espèces.

En analysant les corrélations entre les morphotypes de CMA (tableau V), les analyses statistiques ont révélé des corrélations très fortement significatives au sein du genre Glomus (exemple : corrélation entre Glomus etunicatum et Rhizophagus intraradices ou Rhizophagus irregularis, ou encore entre Rhizophagus intraradices et Rhizophagus irregularis). Le tableau VI révèle que le site d'Amskroud $M$ présentait le plus de corrélations significatives avec d'autres sites, en l'occurrence les sites d'Argana, Bouizakarne ou Essaouira. 


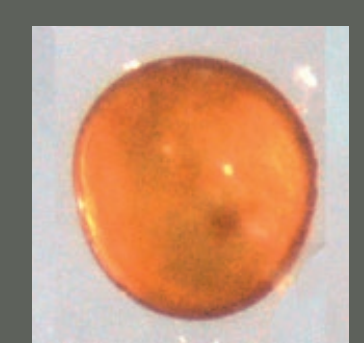

Sclerocystis sinuosum

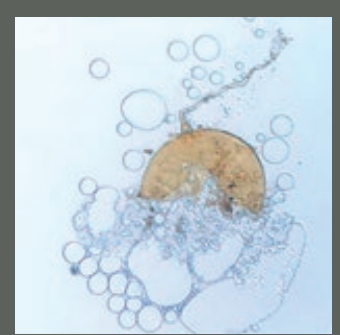

Rhizophagus aggregatus

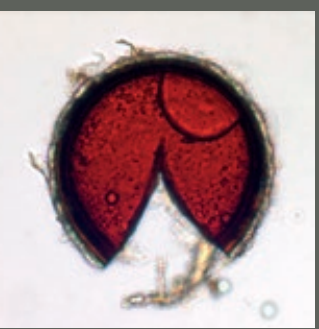

Septoglomus constrictum

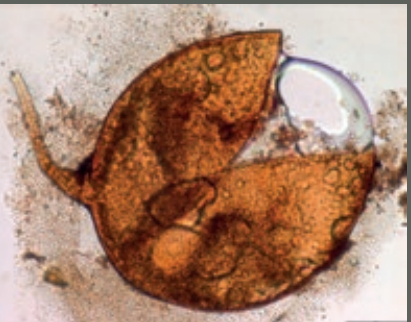

Rhizophagus intraradices

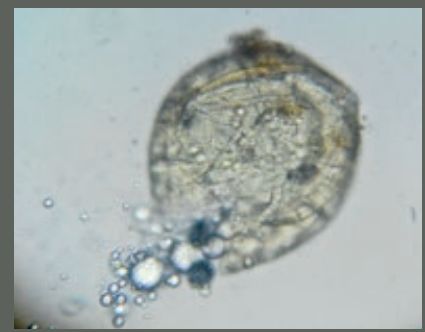

Scutellospora sp1

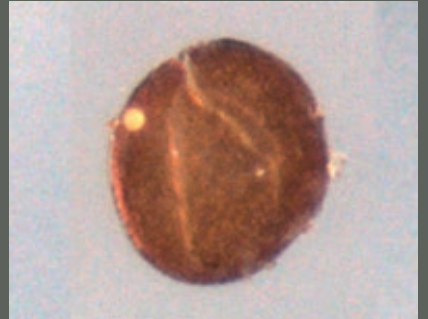

Rhizophagus irregularis

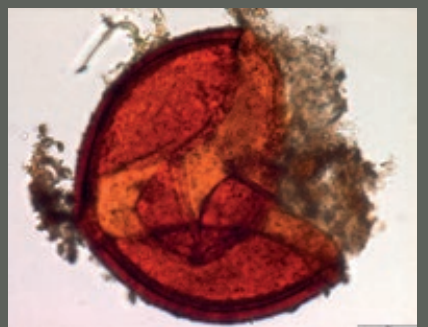

Scutellospora sp2

Figure 4.

Genres de gloméromycètes rencontrés sous arganier à l'état naturel.

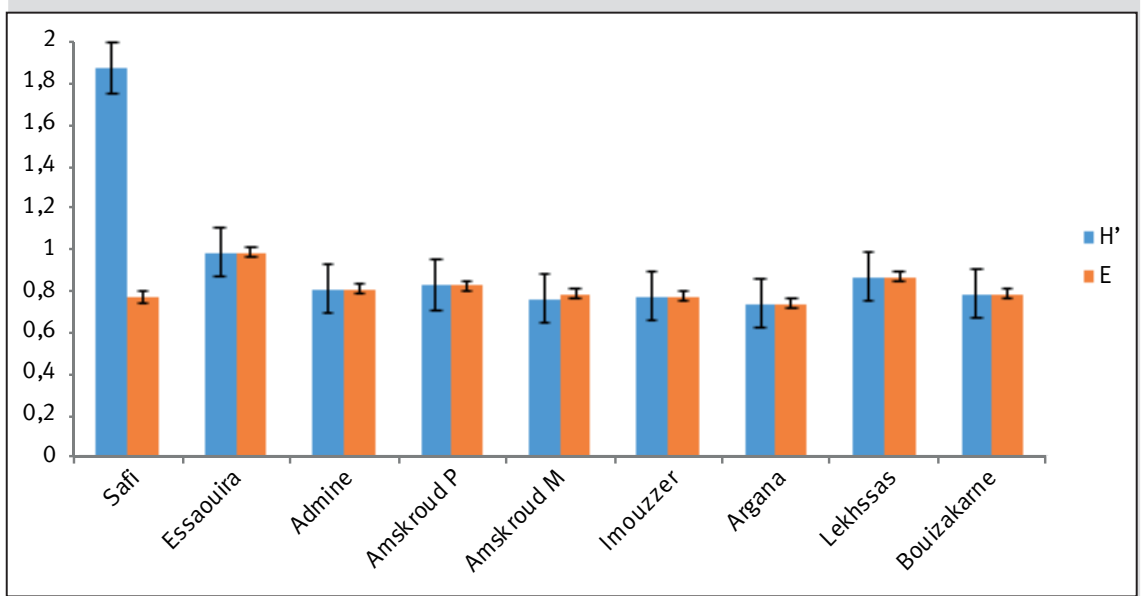

Figure 5.

Indice de Shannon-Wiener ( $\left.\mathrm{H}^{\prime}\right)$ et indice d'équitabilité de

Piélou (E) des neuf stations.

\section{Discussion}

Les sols sont généralement des milieux complexes et particuliers. La perte d'une ou plusieurs de leurs propriétés dégrade leur capacité à produire de la biomasse. Les facteurs environnementaux tels que les caractéristiques physico-chimiques, le $\mathrm{pH}$ et l'humidité du sol influencent les populations microbiennes (Anderson et Domsch, 1993 ; Stotzky, 1997). L'analyse des paramètres physico-chimiques des neuf sols des arganeraies marocaines a été effectuée dans le but de comprendre la répartition, l'abondance et l'efficience des champignons mycorhiziens.

La distribution des fractions minérales montre que les sols récoltés au sein des arganeraies de Lekhssas et Amskroud plaine sont relativement plus riches en argile que celui de Safi, plutôt limonneux, alors que les sols d'Essaouira et Imouzzer sont sablonneux. Selon Brundrett (1991), les sols riches en colloïdes seraient favorables à la formation des mycorhizes. Les sols riches en matière organique peuvent stimuler le fonctionnement des champignons mycorhiziens à arbuscules (Ryan et al., 1994). Les sols de Safi et Amskroud plaine présentent des teneurs élevées en biomasse organique, en raison de leur usage agricole. Les teneurs en $P$ assimilable y sont également plus élevées, ce qui pourrait affecter la production et l'efficacité des champignons mycorhiziens. L'augmentation du phosphore réduit la colonisation fongique, la densité et la diversité des spores (Gosling et al., 2006).

Les sols marocains ont un caractère calco-alcalin (Carle, 1930). Les échantillons rhizosphériques des arganeraies étudiées ont un pH légèrement alcalin (7,12 à 7,62). La solubilité des phosphates et d'autres éléments minéraux y devient difficile, d'où l'intérêt des associations entre plantes et micro-organismes, qui exercent des contrôles rétroactifs par exsudation d'acides organiques, de ligands et d'une gamme de composés qui favorisent la dissolution des minéraux et la libération de nutriments dans des formes disponibles aux plantes (Hinsinger et al., 2011). Parmi ces associations, la symbiose endomycorhizienne à arbuscules est la plus

\section{Potentiel mycorhizogène}

Les sols d'Argana, Bouizakarne et Imouzzer présentaient les potentiels mycorhizogènes les plus élevés, suivis par ceux des sols de Safi et d'Amskroud (plaine et montagne) et ceux de Lekhssas et Essaouira (figure 6). répandue chez les plantes (Corkidi et al., 2008 ; Fortin et al., 2008). L'arganier est connu pour ce type de relation dont le statut a été étudié dans la région du Souss-Massa au Maroc. Plusieurs auteurs confirment la dépendance de cet arbre aux CMA (Achouri, 1989 ; Nouaim et Chaussod, 1994, 2002 ; Bousselmane et al., 2002 ; El Mrabet et al., 2013). Dans notre étude, les fréquences de mycorhization quantifiées ont été 
Tableau V.

Corrélation entre les différents morphotypes de CMA isolés.

\begin{tabular}{|c|c|c|c|c|c|c|c|}
\hline & $\begin{array}{c}\text { Rhizophagus } \\
\text { aggregatus }\end{array}$ & $\begin{array}{l}\text { Funneliformis } \\
\text { constrictum }\end{array}$ & $\begin{array}{l}\text { Sclerocystis } \\
\text { sinuosa }\end{array}$ & $\begin{array}{l}\text { Rhizophagus } \\
\text { intraradices }\end{array}$ & $\begin{array}{c}\text { Rhizophagus } \\
\text { irregularis }\end{array}$ & $\begin{array}{l}\text { Scutellospora } \\
\text { sp1 }\end{array}$ & $\begin{array}{l}\text { Scutellospora } \\
\text { sp2 }\end{array}$ \\
\hline Rhizophagus aggregatus & 1,0000 & $0,1728 \mathrm{~ns}$ & $0,4583 \mathrm{~ns}$ & $0,2391 \mathrm{~ns}$ & $0,5369 \mathrm{~ns}$ & $0,5957 \mathrm{~ns}$ & $0,6756^{\star}$ \\
\hline Funneliformis constrictum & & 1,0000 & $0,0729 \mathrm{~ns}$ & $0,1496 \mathrm{~ns}$ & $0,0319 \mathrm{~ns}$ & $0,0312 \mathrm{~ns}$ & $0,3077 \mathrm{~ns}$ \\
\hline Sclerocystis sinuosa & & & 1,0000 & $0,9191^{\star \star \star}$ & $0,9087^{\star \star \star}$ & $-0,0463 n s$ & $0,1652 \mathrm{~ns}$ \\
\hline Rhizophagus intraradices & & & & 1,0000 & $0,9265^{\star \star \star}$ & $-0,3478 n s$ & $0,1843 \mathrm{~ns}$ \\
\hline Rhizophagus irregularis & & & & & 1,0000 & $0,1864 \mathrm{~ns}$ & $0,1339 \mathrm{~ns}$ \\
\hline Scutellospora sp1 & & & & & & 1,0000 & $0,3602 \mathrm{~ns}$ \\
\hline Scutellospora sp2 & & & & & & & 1,0000 \\
\hline
\end{tabular}

Tableau VI.

Corrélation entre les différents sites étudiés.

\begin{tabular}{|c|c|c|c|c|c|c|c|c|c|}
\hline & Admine & Amskroud M & Amskroud P & Argana & Bouizakarne & Essaouira & Imouzzer & Lekhssas & Safi \\
\hline Admine & 1,0000 & $0,8471^{*}$ & $-0,0145 \mathrm{~ns}$ & $0,8130^{*}$ & $0,3489 \mathrm{~ns}$ & $0,7140 \mathrm{~ns}$ & $0,0852 \mathrm{~ns}$ & $0,0883 \mathrm{~ns}$ & $0,7358 \mathrm{~ns}$ \\
\hline Amskroud M & & 1,0000 & $0,0925 \mathrm{~ns}$ & $0,9674^{\star \star}$ & $0,7821^{\star}$ & $0,9423^{*}$ & $0,1173 \mathrm{~ns}$ & $0,4724 \mathrm{~ns}$ & $0,5052 \mathrm{~ns}$ \\
\hline Amskroud P & & & 1,0000 & $0,0816 \mathrm{~ns}$ & $0,1439 \mathrm{~ns}$ & $-0,0510 \mathrm{~ns}$ & $0,9456^{\star}$ & $0,7333 \mathrm{~ns}$ & $-0,1264 \mathrm{~ns}$ \\
\hline Argana & & & & 1,0000 & $0,7154 \mathrm{~ns}$ & $0,9752^{\star \star}$ & $0,0843 \mathrm{~ns}$ & $0,4127 \mathrm{~ns}$ & $0,5981 \mathrm{~ns}$ \\
\hline Bouizakarne & & & & & 1,0000 & $0,7909^{\star}$ & $0,0907 \mathrm{~ns}$ & $0,7315 \mathrm{~ns}$ & $-0,0523 \mathrm{~ns}$ \\
\hline Essaouira & & & & & & 1,0000 & $-0,0765 n s$ & $0,3860 \mathrm{~ns}$ & $0,4965 \mathrm{~ns}$ \\
\hline Imouzzer & & & & & & & 1,0000 & $0,7100 \mathrm{~ns}$ & $-0,0378 \mathrm{~ns}$ \\
\hline Lekhssas & & & & & & & & 1,0000 & $-0,2252 \mathrm{~ns}$ \\
\hline Safi & & & & & & & & & 1,0000 \\
\hline
\end{tabular}

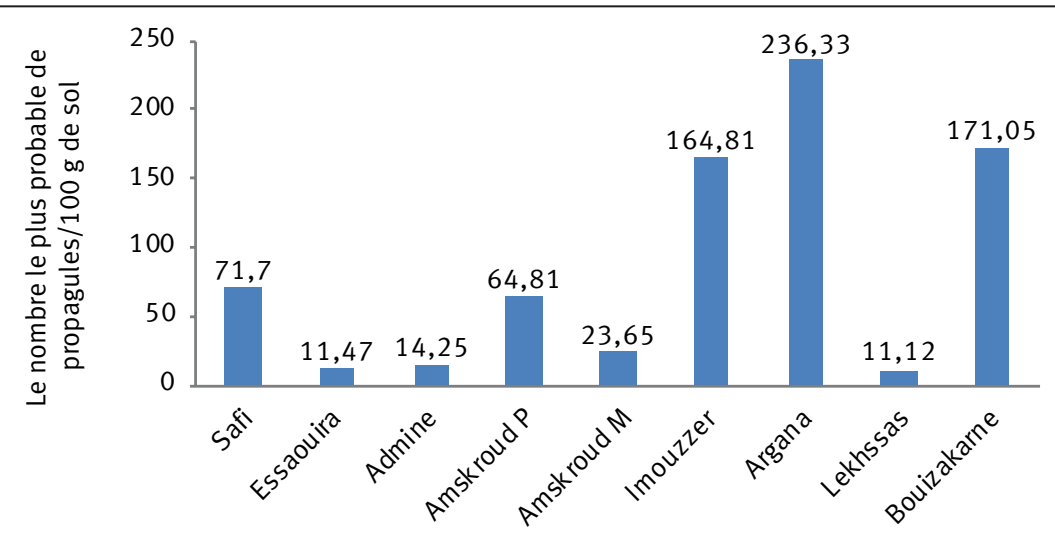

Les sites prospectées

Figure 6.

Potentiel mycorhizogène des sols rhizosphériques de l'arganier. 
élevées dans toutes les arganeraies, ce qui confirme que A. spinosa est une espèce hautement mycotrophe. Pour les autres paramètres de mycorhization, même si l'ANOVA a montré que le facteur site présente une source de variation pour la teneur en arbuscules, la comparaison des moyennes par le test de Tukey ne confirme pas de différenciation significative des sites en teneur de ces mêmes structures. Ceci pourrait être dû à une corrélation négative avec la concentration en $P$ dans les sols rhizosphériques des différentes arganeraies. Les fortes teneurs en phosphore s'avèrent défavorables à la colonisation racinaire (Plenchette et al., 1983 ; Sylvia et al., 1993 ; Garbaye, 2013).

L'isolement des spores de CMA a montré une variabilité intersites de l'abondance et du nombre de morphotypes observés. La densité la plus élevée a été relevée dans le site d'Amskroud plaine (207 spores par $100 \mathrm{~g}$ de sol). Ce nombre reste très faible par rapport à celui rapporté par El Maati et al. (2015), avec 1127 spores par $100 \mathrm{~g}$ dans l'arganeraie d'Ait Baha, mais reste comparable à celui de la rhizosphère d'espèces forestières marocaines comme Tetraclinis articulata (Abbas et al., 2006) ou Cupressus atlantica (Ouahmane et al., 2007).

Les variations morphotypiques des spores fongiques isolées des sols rhizosphériques des différentes arganeraies pourraient traduire la présence d'écotypes dans les sols des sites prospectés. Selon Jeffries et Barea (2001), la nature des communautés de CMA demeure liée au type de peuplement végétal et à l'existence d'écotypes. En outre, une corrélation positive associe la densité des spores à la concentration en matière organique des sols rhizosphériques des différentes arganeraies. Les densités en spores les plus élevées (207 et 102 spores par $100 \mathrm{~g}$ de sol sec) ont été observées dans les sites d'Amskroud plaine et Safi, sites à usage agricole et à teneur élevée en matière organique (13 à $15 \%$ ). L'apport d'amendements organiques (fumier, compost, résidus de culture) peut stimuler la multiplication des CMA (Gosling et al., 2006).

La richesse spécifique en CMA dans les sols rhizosphériques des arganeraies étudiées est de l'ordre de sept morphotypes. Ce faible nombre peut renseigner l'état de dégradation des arganeraies, conformément aux observations réalisées sur d'autres espèces de plantes, dans des environnements arides et semi-arides (Stutz et Morton, 1996 ; Azcón-Aguillar et al., 2003 ; Abbas, 2014).

L'analyse des caractéristiques morpho-anatomiques de la communauté de spores isolée dans notre étude a révélé la présence de deux genres (Glomus et Scutellospora). Kenny et al. (2009) ont noté la présence, dans neuf arganeraies marocaines (Aghroud, Teferdine, Essaouira, Bouzemour, Tamaït, Barrage Abdelmoumen, Ikherdiden, Douar Ighaline, Réserve d'IAV à Agadir), une grande diversité d'espèces endomycorhiziennes. Seules certaines espèces ont été déterminées : Glomus aggregatum, Glomus constrictum, Gigaspora margarita, Entrophospora infrequens. El Maati et al. (2015) ont aussi relevé, dans cinq sites d'arganier (Tizi, Tartatin, Tirhmi, Ait Baha, Tiguert et Alma), la présence de Glomus fasciculatum et d'autres espèces non identifiées appartenant aux genres Glomus (quatre Glomus sp.) et Gigaspora (Gigaspora sp.). Or, Mosse (1973) signale que le genre Glomus apparaît généralement dans les sols à $\mathrm{pH}$ neutre ou alcalin, alors que les genres Gigaspora et Acaulospora tolèrent l'acidité.

Les analyses statistiques ont montré que le site d'Amskroud montagne (Amskroud M) présente plus de corrélations significatives, précisément avec les sites d'Argana, Bouizakarne ou Essaouira. Klironomos et al. (1993) suggèrent que le $\mathrm{pH}$ et la matière organique sont des régulateurs de la sporulation des CMA. Ceci expliquerait en partie les différences de nombre de morphotypes isolés entre les différentes arganeraies marocaines. Mais la dominance du genre Glomus reste commune dans ces zones soumises à la sécheresse.

Les corrélations entre les différents morphotypes de CMA sont très fortement significatives au sein du genre Glomus, prépondérant dans les sols désertiques, probablement parce qu'il contient des isolats adaptés aux fluctuations environnementales (Stutz et al., 2000).

Une corrélation positive apparaît entre la densité de spores et l'intensité de mycorhization des racines de l'arganier dans les sites de Safi et Bouizakarne, avec des densités de spores respectives de 102 et 108 par $100 \mathrm{~g}$ de sol sec, et des intensités de mycorhization de $41 \%$ et $34 \%$. Elle est négative pour Essaouira, Admine et Amskroud plaine. D'autres facteurs édaphoclimatiques ou liés à l'usage du sol peuvent affecter le processus de mycorhization. Les pratiques culturales déterminent significativement la mycorhization : les labours réguliers perturbent la structure du sol et rompent les complexes mycorhiziens (Kabir, 2005), et la monoculture continue et la rotation culturale réduisent le pool de spores des endomycorhizes (Vestberg et al., 2005). De même, les engrais synthétiques perturbent certaines caractéristiques du sol et détruisent les communautés mycorhiziennes (Marschner et al., 2003).

Une corrélation négative lie les paramètres chimiques des sols au potentiel mycorhizogène. Les sols de Safi et d'Ameskroud plaine, à teneurs élevées en carbone total (près de $8 \%$ ), matière organique (près de $14 \%$ ) et phosphore assimilable (près de 54 ppm), ont des PM faibles et donnent lieu à des teneurs en arbuscules plus basses. Par contre, les sols d'Argana, Bouizakarne et Imouzzer, à teneurs intermédiaires en carbone total (près de $4 \%$ ), matière organique (près de $7 \%$ ) et phosphore (près de $27 \%$ ), sont ceux qui ont un fort potentiel mycorhizogène (PM plus élevés). Ces résultats semblent contradictoires avec les paramètres de mycorhization cités auparavant et favorisent plutôt les sols récoltés à Argana et Bouizakarne. Ces différences laissent penser que d'autres facteurs édaphiques (propriétés biologiques des sols) des arganeraies pourraient avoir une grande influence sur la performance des spores mycorhiziennes, et, par suite, induire en erreur une sélection négative de CMA inefficients en matière de survie et de croissance de la plante cible. Des résultats similaires ont été observés par Azcón-Aguilar et al. (2003) et Winding et al. (2005) qui ont montré que le nombre total de spores était relativement bas dans les écosystèmes arides, mais n'était pas corrélé au potentiel mycorhizogène des sols. Ceci ne peut être lié qu'à l'efficacité des spores de CMA dans ces sols, ou à l'intervention du mycélium extra-racinaire des champignons produit par les racines mycorhizées, qui constitue un réseau reliant la plante au sol (Roldan et al., 1994 ; Jeffries et Barea, 2001). 


\section{Conclusion}

La dynamique des végétaux est fortement liée au développement de la composante biotique tellurique, en particulier les micro-organismes symbiotiques. La gestion intégrée des propriétés symbiotiques de l'arganier dans son aire naturelle, par utilisation des ressources microbiennes indigènes, est une voie biotechnologique prometteuse. Elle consiste à rechercher les associations adéquates entre l'arganier et les champignons mycorhiziens afin de réussir les opérations de réimplantation de cette espèce.

La présente étude a été consacrée à l'exploration du statut mycorhizien de l'arganier à l'état naturel au sein de neuf arganeraies marocaines de différentes situations géographiques (montagne, plaine, côtière et continentale), afin d'analyser la diversité morpho-anatomique des champignons endomycorhiziens qui leur est associée in situ et d'évaluer le potentiel mycorhizogène de ces symbiotes fongiques.

Les résultats sur le statut mycorhizien de l'arganier permettent de retenir qu'en milieu naturel l'endomycorhization est une réalité. Tous les fragments de racines analysés sont densément mycorhizés. L'analyse de la richesse spécifique en spores de CMA montre que les rhizosphères des arganeraies prospectées abritent des communautés de CMA variées dominées par le genre Glomus. Les sols récoltés au niveau d'Imouzzer, Argana et Bouizakarne ont présenté les meilleures intensités de colonisation racinaire. Les différences constatées au sein des arganeraies prospectées suggèrent que le choix des symbioses mycorhiziennes les plus efficaces dépend des facteurs édaphoclimatiques et des pratiques culturales.

Si la symbiose mycorhizienne constitue un élément clé dans le fonctionnement durable des écosystèmes végétaux terrestres, son application pour l'espèce Argania spinosa doit être optimisée en prenant en considération les exigences du milieu à régénérer ou à réhabiliter.

\section{Remerciements}

Nous remercions l'ANDZOA, Agrotech et Lafarge pour leur soutien financier accordé aux doctorants dans le cadre des bourses « Jeunes Chercheurs de l'arganier».

\section{Références bibliographiques}

Abbas Y., Ducousso M., Abourough M., Azcon R., Duponnois R., 2006. Diversity of arbuscular mycorrhizal fungi in Tetraclinis articulata (Vahl) Master woodlands in Morocco. Annals of Forest Science, 63: 285-291. https://doi10.1051/ forest:2006007

Abbas Y., 2014. Microorganismes de la rhizosphère des Tétraclinaies : un outil pour optimiser la régénération assistée du Tetraclinis articulata (Vahl.) Master. Thèse de Doctorat National, Université Mohamed V, Faculté des Sciences, Rabat - Maroc. 57-80. https://toubkal.imist.ma/ handle/123456789/10002

Abourouh M., 1992. Essai de mycorhization en pépinière par les spores de Pisolithus tinctorius. Annales de la recherche forestière au Maroc, 26 : 126-137.

Abourouh M., 2007. La recherche scientifique sur l'arganier: bilan et perspectives. Annales de la Recherche Forestière au Maroc, $38:$ 22-31.

Achouri M., 1989. Endogonaceae of Souss Massa, Morocco. PhD Thesis, university of Minnesota, USA. 121.

Alexander M., 1965. Most probable number method for microbial populations.In: C. A. Black et al. (ed.), Methods of soil analysis, part 2. American Society Agronomy, Madison, 1467-1472.

Alguacil M. M., Torrecillas E., Kohler J., Roldan A., 2011. Amolecular approach to ascertain the success of "in situ" AM fungi inoculation in the revegetation of a semiarid, degraded land. Science of the Total Environment, 409: 2874-2880. https://doi.org/10.1016/i.scitotenv.2011.04.029

Anderson T. H., Domsch K. H., 1993.The metabolic quotient for $\mathrm{CO} 2$ (q $\mathrm{CO} 2$ ) as a specific activity parameter to assess the effects of environmental conditions such as $\mathrm{pH}$, on the microbial biomass of forest soils. Soil Biology \& Biochemistry, 25: 393-395.

Anne A., 1945. Dosage rapide du carbone organique des sols. Annals of Agriculture, 2: 100-105.

Azcón-Aguilar C., Palenzuela J., Roide A., Bautista S., Vallejo R., Barea J. M., 2003. Analysis of the mycorrhizal potential in the rhizosphere of representative plant species from desertificationthreaned Mediterranean shrublands. Applied Soil Ecology, 22: 29-37. https://doi.org/10.1016/ S0929-1393(02)00107-5

Benabid A., 2000. Flore et écosystèmes du Maroc. Édition Ibis Press Paris, 335 p.

Bousselmane F., Kenny L., Achouri M., 2002. Effet des mycorhizes à vésicules et arbuscules sur la croissance et la nutrition de l'arganier (Argania spinosa L.).Actes Inst. Agron. Vet. (Maroc), 22 (4): 193-198.

Brundrett M. C., 1991. Mycorrhizas in natural ecosystems. In: Macfayden A., Begon M., Fitter A. H. (Eds), Advances in Ecological Research. Academic Press Ltd., London, 21: 171313. https://doi.org/10.1016/S0065-2504(08)60099-9

Brundrett M., Bougher N., Dell B., Grove T., Malajczuk N., 1996. Working with Mycorrhizas in Forestry and Agriculture. ACIAR Monograph 32. http://irt-pw-cp1.irt.csus.edu/jbaxter/wp-content/uploads/2016/02/1996-Brundrett-et-alMycorrhizal-Methods-Book.pdf 
Carle G., 1930. Étude sur les Sols marocains (Suite et fin). Revue de botanique appliquée et d'agriculture coloniale, $10^{\mathrm{e}}$ année, 102 : 91-99. https://doi.org/10.3406/ jatba.1930.4828

Cochran W. G., 1950.Estimation of bacterial densities by means of the "most probable number." Biometrics, 6:105116. https://www.jstor.org/stable/3001491

Corkidi L., Evans M., Bohn J., 2008. An introduction to propagationof arbuscular mycorrhizal fungi in pot cultures for inoculation of native plant nursery stock. Native plants Journal, 9:29-38. http://npj.uwpress.org/content/9/1/29.short El Maati Y., Msanda F., El Mousadik A., El Hamdaoui A., El Mrabet S., Ouahmane L., 2015. Contribution to the characterization of mycorrhizae in the south west of Morocco and their effect on growth parameters of Argania spinosa. The American Journal of Innovative Research and Applied Sciences, 1 (7): 235-243. https://www.researchgate.net/ profile/Elmaati Yassine/publication/282975582 CONTRIBUTION TO THE CHARACTERIZATION OF MYCORRHIZAE IN THE SOUTH WEST OF MOROCCO AND THEIR EFFECT ON GROWTH PARAMETERS OF ARGANIA SPINOSA/ links/5628c15908ae22b1702ed227/CONTRIBUTION-TOTHE-CHARACTERIZATION-OF-MYCORRHIZAE-IN-THE-SOUTHWEST-OF-MOROCCO-AND-THEIR-EFFECT-ON-GROWTH-PARAMETERS-OF-ARGANIA-SPINOSA.pdf

El Mrabet S., Ouahmane L., El Mousadik A., Msanda F., Abbas Y., 2013. L'éfficacité de l'inoculation mycorhyzienne et de l'addition du bio-compost sur le développement d'Argania spinosa sur le champ. Actes du 2 ème congrès international de l'Arganier, Maroc, 47-58.

Fisher R. A., Yates F., 1970. Statistical Tables for Biological Agriculture and Medical Research, sixth ed. Hafner Publishing Company, Davien. 1938 p. https://www.cabdirect.org/ cabdirect/abstract/19391601696

Fortin J. A., Plenchette C., Piché Y., 2008. Les mycorhizes : La nouvelle révolution verte. Éditions Multimondes.131. http:// www.worldcat.org/title/mycorhizes-lessor-de-la-nouvellervolution-verte/oclc/1009074158

Garbaye J., 2013. La symbiose mycorhizienne, une association entre les plantes et les champignons. Éditions Quæ, Versailles, 251 p. http://www.worldcat.org/title/symbiose-mycorhizienne-une-association-entre-les-plantes-et-l es-champignons/oclc/1003601944

Gerdeman J. W, Nicolson T. H., 1963. Spore of mycorrhizal endogone species extracted from soil by wet sieving and decanting. Transactions of the British Mycological Society, 46: 235-244. https://doi.org/10.1016/ S0007-1536(63)80079-0

Gosling P., Hodge A., Goodlass G., Bending G. D., 2006. Arbuscular mycorrhizal fungi and organic farming.Agriculture, Ecosystems and Environment, 113: 17-35. https://doi. org/10.1016/i.agee.2005.09.009

Hinsinger P., Brauman A., Devau N., Gérard F., Jourdan C., Laclau J. P., et al., 2011. Acquisition of phosphorus and other poorly mobile nutrients by roots. Where do plant nutrition models fail? Plant and Soil, 348: 29-61. https://doi. org/10.1007/s11104-011-0903-y

Jeffries P., Barea J. M., 2001. Arbuscular mycorrhiza: a key component of sustainable plant-soil ecosystems. In: Hock B. (Ed), The Mycota: Vol. IX Fungal Associations. Springer, Berlin, 95-113. https://doi.org/10.1007/978-3-662-07334-6 6
Kabir Z., 2005. Tillage or no-tillage: impact on mycorrhizae. Canadian Journal of Plant Science, 85:23-29. https://doi. org/10.4141/P03-160

Kenny L., Galiana A., Bellefontaine R., 2009. Projet UE/ MEDA/ADS. Appui à l'amélioration de la situation de l'emploi de la femme rurale et gestion durable de l'arganeraie dans le sud-ouest du Maroc - Thème 2 : Multiplication végétative et symbioses racinaires de l'arganier : Optimisation des agrosystèmes à base d'arganier. Agence du développement social (Maroc) et Agropolis (France), Rapport final, 1-71.

Klironomos J. N., Moutoglis P., Kendrick B., Widden P., 1993. A comparison of spatial heterogeneity of vesicular arbuscular mycorrhizal fungi in two maple-forest soils. Canadian Journal of Botany, 71:1472-14807. https://doi.org/10.1139/ b93-178

Koske R. E., Tessier B., 1983. A convenient permanent slide mounting medium, Mycological Society of America Newsletter, 34: 59.

Lachapelle J., 2004. Microscopie, méthodes d'examen, colorations. Revue du Cercle de Mycologie de Bruxelles, 4 : 35-71. http://www.cercle-myco-bruxelles.be/Publications/04/Microscopie\%20methodes\%20colorations.pdf

Lahcen K., Galiana A., Bellefontaine R., 2009. Appui à l'amélioration de la situation de l'emploi de la femme rurale et gestion durable de l'arganeraie dans le sud-ouest du Maroc - Thème2 : Multiplication végétative et symbioses racinaires de l'arganier Optimisation des agro systèmes à base d'arganier.Projet UE / MEDA / ADS, Rapport final, 71 p.

M'hirit O., Benzyane M., Benchekroun F., El Yousfi S. M., Bendaanoun M., 1998. L'arganier. Une espèce fruitière-forestière à usages multiples. Sprimont, Belgique, Mardaga. 151p. Malusá E., SasPaszt L., Głuszek S., Ciesielska J., 2014. Organic Fertilizers to Sustain Soil Fertility. In: Sinha S., Pant K. (eds), Fertilizers Technology Vol. 1: Synthesis. Publishers Studium Press LLC, Houston, USA, 256-281.

Marschner P., Kandeler E., Marschner B., 2003. Structure and Function of the Soil Microbial Community in a Long-Term Fertilizer Experiment.Soil Biology and Biochemistry, 35: 453461. https://doi.org/10.1016/S0038-0717(02)00297-3

Meriaux S., 1954. Contribution à l'étude de la granulométrie. Thèse d'État, Paris, 118 p.

Morton J. B., Benny G. L., 1990. Revised classification of arbuscular mycorrhizal fungi (Zygomycetes): a new order, Glomales, two new suborders, Glominae and Gigasporinae, and two new families, Acaulosporaceae, with an emendation of Glomaceae. Mycotaxon, 37: 471-491. https://www. cabdirect.org/cabdirect/abstract/19912307931

Mosse B., 1973. Advances in the study of vesicular-arbuscular mycorrhiza. Annual Review of Phytopathology, 11: 171-196. https://doi.org/10.1146/annurev. py.11.090173.001131

Mosse B., 1986. Mycorrhiza in a sustainable agriculture. Biological Agriculture and Horticulture, 3: 191-209. https:// doi.org/10.1080/01448765.1986.9754471

Nouaim R., Chaussod R., El Aboudi A., Schnabel C., Peltier J. P., 1991. L'Arganier. Essai de synthèse des connaissances sur cet arbre. In: Physiologie des arbres et arbustes en zones arides et semi-arides. Groupe d'Étude de l'Arbre, 373-388.

Nouaim R., Chaussod R., 1994. Mycorrhizal dependency of two clones of micropropagatedArgan tree (Argania spinosa): l) Growth and biomass production. Agroforestry Systems, 27: 53-65. https://doi.org/10.1007/BF00704834 
Nouaim R., Chaussod R., 1997. Effet de la mycorhizationcontrolée sur la croissance de l'arganier (Argania spinosa) après sa transplantation en sol non désinfecté. Al Awamia, 96 : 65-76. http://webagris.inra.org.ma/doc/awamia/09608.pdf

Nouaim R., Chaussod R., 2002., Réponse à la mycorhization de plants d'arganier (Argania spinosa) multipliés par bouturage. Al Alwamia, 105 : 9-22.

Olsen S. R., Cole C. V., Watanabe F. S., Dean L. A. 1954.Estimation of available phosphorus in soils by extraction with sodium bicarbonate. Washington, DC: US Department of Agriculture, Circular, 939: 19. http://krishikosh.egranth. ac.in/bitstream/1/2041713/1/41717.pdf

Ouahmane L., Hafidi M., Thioulouse J., Ducousso M., Kisa M., Prin Y., et al., 2007. Improvement of Cupressus atlantica Gaussen growth by inoculation with native arbuscular mycorrhizal fungi.Journal of Applied Microbiology, 103: 683690. https://doi.org/10.1111/i.1365-2672.2007.03296.x

Philips J.M., Hayman D.S., 1970. Improved procedures for clearing roots and staining parasitic and vesicular arbuscular mycorrhizal fungi for rapid assessment of infection.Transactions of the British Mycological Society, 55: 158-161.

Plenchette C., Fortin J. A., Furlan V., 1983. Growth responses of several plant species to mycorrhizae in a soil of moderate $P$ fertility: I. Mycorrhizal dependency under field conditions. Plant Soil, 70: 199-209. https://doi.org/10.1007/ $\underline{B F 02374780}$

Rillig M.C., Mummey D.L., 2006. Mycorrhizas and soil structure. New Phytologist, 171: 41-53. https://doi. org/10.1111/j.1469-8137.2006.01750.x

Roldan A., García-Orenes F., Lax A., 1994. An incubation experiment to determine factors involving aggregation changes in an arid soil receiving urban refuse. Soil Biology \& Biochemistry, 26: 1699-1707. https://doi. org/10.1016/0038-0717(94)90323-9

Ryan M. H., Chilvers G. A., Dumaresq D. C., 1994. Colonisation of wheat by VA mycorrhizal fungi was found to be higher on a farm managed in an organic Matter than on a conventional neighbour. Plant and Soil, 160: 33-40. https://doi. org/10.1007/BF00150343

Sanon A. A., 2005. Rôle des champignons mycorhiziens à arbuscules dans les mécanismes régissant la co-existence entre espèces végétales. D.E.A. National de SCIENCE DU SOL, Nancy, France, 4-6. http://horizon.documentation.ird. fr/exl-doc/pleins textes/divers13-07/010040027.pdf

Schmid T., Meyer J., Oehl F., 2008. Integration of mycorrhizal inoculum in high alpine revegetation. In: Feldmann F., Kapulnik Y., Baar J.(eds), Mycorrhiza Works.. Proceedings of the International Symposium "Mycorrhiza for Plant Vitality" and the Joint Meeting of Working Groups 1 - 4 of COST Action 870. Deutsche Phytomedizinische Gesellschaft, Braunschweig, Germany, 278-288. https://www.cabdirect.org/cabdirect/ abstract/20153030200

Sieverding E., 1991. Vesicular-arbuscular mycorrhiza management in tropical agrosystems. Deutsche Gesell schaft fürTechnische Zusammen arbeit (GTZ), Eschborn, Germany, 371p.

Smith S.E., Read D.J., 2008. Mycorrhizal symbiosis, 3rd edition. Waltham, MA, USA: Academic Press.
Stotzky G., 1997. Soil as an environment for microbial live. In: Van ElsasJ. D., Trevorset J. T., WellingtonE. M. H.(eds), Modern Soil Microbiology. Dekker, New York, 1-20.

Stutz J.C., Morton J.B., 1996. Successive pot cultures reveal high species richness of arbuscular mycorrhizal fungi in arid ecosystems. Canadian Journal of Botany, 74: 1883-1889. https://doi.org/10.1139/b96-225

Stutz J.C., Coperman R., Martin C.A., Morton J.B., 2000. Patterns of species composition and distribution of arbuscular mycorrhizal fungi in arid regions of southwestern North America and Namibia, Africa. Canadian Journal of Botany, 78: 237-245. https://doi.org/10.1139/b99-183

Sylvia D.M., Wilson D.O., Graham J.H., Maddox J.J., Millner P., Morton J.B., et al., 1993. Evaluation of vesicular arbuscular mycorrhiza fungi in diverse plants and soil. Soil Biology and Biochemistry, 25 (6): 705- 713. https://pubag.nal.usda. gov/catalog/35691

Tilman D., Downing J.A., 1994. Biodiversity and stability in grasslands. Nature, 367: 363-365. https://10.1038/367363a0

Trouvelot A., Kough J. L., Gianinazzi V., 1986. Mesure de taux de mycorhization VA d'un système radiculaire. Recherche de méthodes d'estimation ayant une signification fonctionnelle. In: Gianinazzi-Pearson V., Gianinazzi S. (eds), Physiological and genetic aspects of mycorhizical. INRA, Paris, 217-221.

Vestberg M., Saari K., Kukkonen S., Hurme T., 2005. Mycotrophy of corps in rotation and soil amendment with peat influence the abundance and effectiveness of indigenous arbuscular mycorrhizal fungi in field soil. Mycorrhiza, 15 (6): 447-458. https://doi.org/10.1007/s00572-005-0349-2

Winding A., Hund-Rinke K., Rutgers M., 2005. The use of microorganisms in ecological soil classification and assessment concepts.Ecotoxicology and Environmental Safety, 62 (2): 230-248. https://doi.org/10.1016/i. ecoenv.2005.03.026

Bois et Forêts des Tropiques - Revue scientifique du Cirad
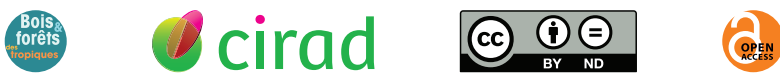

Cirad - Campus international de Baillarguet, 34398 Montpellier Cedex 5, France - Contact : bft@cirad.fr - ISSN : L-0006-579X 